Results 1496 patients were studied: median age 62 (IQR 5173), 58\% female. Median EDS score was 4 (IQR 2.5-6); with $67 \%$ having an EDS $\geq 3.5$. $64 \%$ were triaged to $2 \mathrm{WW}$ endoscopy; $18 \%$ to urgent (but not $2 \mathrm{WW}$ ) endoscopy; $2.8 \%$ to urgent CT scan; $5.5 \%$ to routine OGD; and $4.4 \%$ to barium swallow. After excluding patients who declined investigation, results were available for $96 \%$.

91 UGI cancers were diagnosed (prevalence 7.1\%); with 3 (3\%) UGI cancers diagnosed in patients with $\mathrm{EDS}<3.5$ (one with EDS 3, two with EDS 1.5). EDS $\geq 3.5$ had sensitivity of $96.7 \%$ and negative predictive value of $99 \%$ for UGI cancer. The factors associated with UGI cancer and hence selected to develop CDS included: age odds ratio 1.05 (95\% CI 1.03-1.06); male 3.95 (2.36-6.58); progressive dysphagia 2.30 (1.39-3.79); unintentional weight loss 3.28 (2.02-5.31); acid reflux symptoms $0.47(0.25-0.88)$ and dysphagia localised to the neck $(0.26(0.12-0.57)$. Dysphagia duration less than 6 months was not statistically significant $(1.02 \quad(0.45-$ 2.22). AUROC was 0.83 for CDS as compared to 0.81 for EDS.

Cancer dysphagia score and its receiver operating curve in comparison to Edinburgh dysphagia score is presented in figure 1.

Conclusion A national prospective cohort confirms that EDS has high sensitivity and negative predictive value for UGI cancer, however, a modified cancer dysphagia score offers higher diagnostic accuracy. Our results suggest that CDS should be incorporated in the UGI cancer $2 \mathrm{WW}$ pathway for risk stratification of patients with dysphagia and further studies in primary care are needed.

\section{OTU-13 EUS CHOLEDOCHODUODENOSTOMY IN MALIGNANT DISTAL BILIARY OBSTRUCTION: MULTI-CENTRE COLLABORATION FROM THE UK AND IRELAND}

\begin{abstract}
'Wei On*, 'Bharat Paranandi, 'Andrew M Smith, 'Alistair Young, 'James Pine, ${ }^{2}$ Suresh V Venkatachalapathy, ${ }^{2}$ Martin W James, ${ }^{2}$ Guruprasad P Aithal, ${ }^{2}$ loannis Varbobitis, ${ }^{3}$ Danny Cheriyan, ${ }^{3}$ Ciaran McDonald, ${ }^{4}$ John $S$ Leeds, ${ }^{4}$ Manu Nayar, ${ }^{4}$ Kofi Oppong, ${ }^{5}$ Joe Geraghty, ${ }^{6}$ John Devlin, ${ }^{6}$ Wafaa Ahmed, ${ }^{7}$ Ryan Scott, ${ }^{8}$ Terence Wong, ${ }^{1}$ Matthew T Huggett. 'Leeds Teaching Hospitals NHS Trust, Leeds, UK; ${ }^{2}$ Nottingham Digestive Diseases Centre (NDDC) and NIHR Nottingham Biomedical Research Centre (BRC), Nottingham University and Nottingham University Hospitals NHS Trust, Queen's Medical Centre, Nottingham, UK; ${ }^{3}$ Beaumont Hospital and RCSI, Dublin, Ireland; ${ }^{4}$ The Newcastle upon Tyne Hospitals NHS Foundation Trust, Newcastle, UK; ${ }^{5}$ Manchester University NHS Foundation Trust, Manchester, UK; ${ }^{6}$ King's College Hospital NHS Foundation Trust, London, UK; 'Belfast Health and Social Care Trust, Belfast, Ireland; ${ }^{8}$ Guys' and St Thomas' NHS Foundation Trust, London, UK
\end{abstract}

\subsection{6/gutjnl-2021-BSG.3}

Introduction Endoscopic ultrasound guided choledochoduodenostomy (EUS-CDD) with electrocautery enhanced lumen apposing metal stents (EC-LAMS) has emerged as a viable option in the management of patients with malignant distal biliary obstruction (MDBO). We conducted a multi-centre collaboration from the UK and Ireland with an aim to analyse the pooled efficacy, safety and long term outcomes of EUS-CDD for treatment of MDBO.

Methods Consecutive patients with MDBO who underwent EUS-CDD with EC-LAMS at 8 tertiary hepatopancreatobiliary institutions between September 2016 and September 2020 were retrospectively analysed. Recorded variables included patient demographics, procedural characteristics and follow-up data.

\begin{tabular}{|c|c|}
\hline $\begin{array}{l}\text { Severity of } \\
\text { adverse event }\end{array}$ & Adverse event description \\
\hline Mild $(n=6)$ & $\begin{array}{l}\text { - Cholangitis with no stent occlusion treated with antibiotics ( } n=5) \\
\text { - Pneumoperitoneum post stent deployment; bridged with metal } \\
\text { stent with no post-procedural clinical consequence }(n=1)\end{array}$ \\
\hline Moderate $(n=11)$ & $\begin{array}{l}\text { - Cholangitis and stent occlusion requiring re-intervention }(n=6) \\
\text { - Stent migration requiring re-intervention }(n=2) \\
\text { - Stent occlusion without cholangitis requiring re-intervention }(n=1) \\
\text { - Bile leak due to malposition of stent requiring re-intervention } \\
(n=1) \\
\text { - Bile leak due to maldeployment of stent; managed conservatively } \\
(n=1)\end{array}$ \\
\hline Severe $(n=2)$ & - Duodenal perforation requiring laparotomy $(n=2)$ \\
\hline
\end{tabular}

Results 112 patients (59 male) were identified. The mean age was 72 years old (range 46 - 94 years old). Pancreatic adenocarcinoma was the commonest underlying malignancy in $63.4 \%(n=71)$. The indications for EUS-CDD were: an inaccessible papilla $(n=49)$, tumour infiltration of papilla $(n=29)$ and unsuccessful biliary cannulation/stricture impassable with guidewire $(n=34)$. Technical success was achieved in $91.1 \%$ $(n=102)$ of patients. Additional stenting with plastic pigtails through the EC-LAMS was performed in 26 patients at the discretion of the endoscopist to augment biliary drainage. Data for clinical success (reduction of serum bilirubin to $\leq 50 \%$ of original value at day 7) was available for 90 patients and was achieved in $94.4 \%$ of these $(n=84)$. The adverse event rate was $16.9 \%(n=19)$ and further details are summarised in the table. The biliary re-intervention rate was $8.1 \%$ $(n=8)$ in 99 patients with successful EUS-CDD (3 lost to follow-up), over a median follow-up of 70 days (range $3-761$ days). Eight patients underwent attempted surgical resection of their primary tumour and in those who did, resection and formation of hepaticojejunostomy was successful.

Conclusion We present the first collaborative data from the UK and Ireland demonstrating EUS-CDD in MDBO to be efficacious with a reasonable safety profile.

\section{OTU-14 COLONOSCOPY POLYP DETECTION IS LOWER AT WEEKENDS: A NATIONAL ENDOSCOPY DATABASE ANALYSIS}

${ }^{1}$ Liya Lu, ${ }^{1,2}$ Jamie Catlow, ${ }^{3}$ Raphael Broughton, ${ }^{4}$ Peter Rogers, ${ }^{1}$ Linda Sharp, 1,2 Matt Rutter*. 'Population Health Sciences Institute, Newcastle University, on behalf of the NED-APRIQOT Team; ${ }^{2}$ North Tees NHS Foundation Trust; ${ }^{3} J A G, R C P$ London; ${ }^{4}$ Weblogik Ltd

\subsection{6/gutjnl-2021-BSG.4}

Introduction Due to the mismatch between endoscopy capacity and demand, many centres use in-reach services at weekends. We used data from the National Endoscopy Database to investigate whether (1) patient characteristics on weekdays and weekends differ and (2) weekend procedures are associated with lower polyp detection, after accounting for patient characteristics.

Methods We conducted a retrospective cross-sectional study using data on independent colonoscopies conducted 01/01-04/ $04 / 2019$. Procedures had to be non-emergencies, undertaken on patients aged $\geq 18$ years, involve complete examinations 